Konrad Reinhart, Carolin Fleischmann-Struzek und Heiko Herwald

\title{
Sepsis und die COVID-19-Pandemie: Herausforderungen und Chancen für die Weiterentwicklung des Gesundheitssystems in Deutschland
}

\begin{abstract}
Sepsis and the COVID-19 Pandemic: Challenges and Chances for Developing a Better Health System in Germany. Sepsis is the most serious complication of infectious diseases including patients critically ill with COVID-19. In 2017, sepsis was estimated to affect close to 50 million people and to cause or contribute to 11 million deaths, with over 3 million of those deaths being in children or adolescents. The WHO considers that most deaths from sepsis are preventable and therefore urges all member states to integrate sepsis in their national health strategy. However, this is not yet the case for Germany, although - compared to other western industrial countries - sepsis mortality rates in Germany are very high. The COVID-19 pandemic has presented the world with the most serious health threat in living memory, which until July 2021 resulted in more than 4 million deaths and unprecedented social and economic disruption. It has reminded us that infectious diseases still present a major global health threat. In contrast to the poor recognition of sepsis from other infections, the response to the pandemic has also been unprecedented in most countries in terms of instituting effective public health measures, and the global scientific community has come together to produce robust research evidence and novel vaccines in record time. Thus, a positive legacy for the COVID-19 pandemic in Germany and around the globe would reduce the global burden of sepsis by making pandemic preparedness, infection prevention and control, sepsis and antimicrobial resistance integral parts of national infection control and management strategies.
\end{abstract}

\section{Einleitung}

Die COVID-19-Pandemie wird als größte Krise seit dem 2. Weltkrieg bezeichnet. Sie hat die Weltgemeinschaft völlig unvorbereitet getroffen - obwohl die sogenannte

\footnotetext{
Konrad Reinhart, Charité - Universitätsmedizin Berlin; Global Sepsis Alliance; Sepsis-Stiftung Carolin Fleischmann-Struzek, IFB Sepsis und Sepsisfolgen, Universitätsklinikum Jena Heiko Herwald, Division of Infection Medicine, Biomedical Center (BMC), Lund University

๑ OpenAccess. ๑ 2021 Konrad Reinhart, Carolin Fleischmann-Struzek und Heiko Herwald, publiziert von De Gruyter. (c) Br-NC-SA Dieses Werk ist lizenziert unter einer Creative Commons Namensnennung - Nicht kommerziell - Weitergabe unter gleichen Bedingungen 4.0 International Lizenz. https://doi.org/10.1515/9783110713336-030
} 
„Spanische Grippe“, die zwischen 1918/19 weltweit ca. 50 Millionen Menschenleben kostete, erst relativ kurz zurückliegt. Die 1896 von Sir William Osler getroffene Feststellung, dass die Menschheit mit Fieber, Hunger und Krieg drei große Feinde habe - von denen mit großem Abstand der schrecklichste das Fieber sei, trifft immer noch zu. Die Unterschätzung der Bedrohung durch Infektionskrankheiten in der heutigen Zeit betrifft nicht nur die Gefahren durch die periodisch auftretenden Endemien und Pandemien, sondern zahlenmäßig in weit höherem Ausmaß die permanente gesundheitliche Bedrohung durch die schweren Verlaufsformen alltäglicher Infektionen, wie z. B. von Lungenentzündungen, Harnwegsinfekten oder der saisonalen Grippe, die medizinisch als Sepsis und umgangssprachlich oft als „Blutvergiftung“ bezeichnet werden. Zur Sepsis kommt es, wenn eine Infektion durch die körpereigenen Abwehrsysteme nicht mehr lokal begrenzt werden kann. Dies hat zur Folge, dass sich eine überschießende Immunantwort als Kettenreaktion im ganzen Körper ausbreitet. Diese gegen die weitere Ausbreitung und Tötung der Infektionserreger gerichteten Abwehrreaktionen schädigen jedoch auch die körpereignen Organe und führen oft zu einem tödlichen Mehrfach-Organversagen. Die im Rahmen einer Sepsis ausgelöste Immunantwort ist durch den Begriff „Zytokin-Sturm“ unzureichend beschrieben, denn sie umfasst nicht nur den Anstieg der als Zytokine bezeichneten Botenstoffe, sondern auch die direkt gegen die Erreger gerichtete zelluläre Immunantwort und die Aktivierung des sogenannten Komplementsystems, das eng an die Aktivierung des Gerinnungssystems gekoppelt ist (Singer et al. 2016). Laut der Weltgesundheitsorganisation (WHO) können nicht nur Bakterien, Pilze und Protozoen (tierische Einzeller) zu einer Sepsis führen, sondern auch virale Infektionen wie das saisonale Grippevirus und hochinfektiöse Viren wie Corona-, Ebola-, Schweinegrippe- und Vogelgrippe-Viren (World Health Organization 2017). Auch bei schweren Verlaufsformen von COVID-19 handelt es sich um eine Sepsis (World Health Organization Headquarters 2020).

Sepsis trug 2017 mit jährlich ca. 11 Millionen Todesfällen zu 20\% aller weltweiten Todesfälle bei (Rudd et al. 2020). Sie betrifft dabei nicht nur einkommensschwache Länder. In den USA bestand bei 35\% der im Krankenhaus verstorbenen Patienten ein enger Zusammenhang mit einer Sepsis (Rhee et al. 2017), und in Deutschland wurden 2015 bei 18\% der im Krankenhaus Verstorbenen eine Sepsis registriert (Fleischmann-Struzek et al. 2018). Nach jüngsten Schätzungen betragen in den USA die jährlichen Kosten für die Behandlung von Sepsis ca. 62 Milliarden USD (Buchman et al. 2020). Im vorliegenden Beitrag werden die Gründe diskutiert, wieso die Bedrohung durch Infektionserkrankungen derart aus dem kollektiven Bewusstsein der modernen Industriegesellschaften verschwunden ist und welche Konsequenzen aus der derzeit unzureichenden Ausrichtung unseres Gesundheitssystems auf diese Herausforderungen zu ziehen sind. 


\section{Frühere Erfolge im Kampf gegen Infektionskrankheiten sorgten lange für Nachlässigkeit}

Noch bis Ende der 1950er Jahre war die Bedrohung durch Infektionskrankheiten durch persönliche Krankheits- und Verlusterfahrungen unserer Eltern- und Großelterngeneration tief im öffentlichen Bewusstsein verwurzelt. Die Schicksalsschläge durch schwere Verläufe von Infektionskrankheiten wurden in der Bevölkerung als gemeinsame Erfahrung geteilt. Infektionskrankheiten waren alltäglich, etwa in Form von Lungenentzündungen, Kindbettfieber oder Wundund Bauchfellentzündungen, hinzu kam noch die ubiquitäre Durchseuchung mit Tuberkulose oder Masern. Auch die periodisch auftretenden Verwüstungen infolge von Pest, Cholera und anderen Epidemien waren im allgemeinen Bewusstsein präsent. Folgerichtig war das gesamte Gesundheitswesen stark auf Seuchenvermeidung und Behandlung von Infektionskrankheiten ausgerichtet. Dies bedeutete auch eine hohe Priorität seitens der Politik, die sich u.a. in einem starken Öffentlichen Gesundheitsdienst manifestierte. Diese Strategie führte z. B. in den USA im Zeitraum 1900 bis 1950 zu einem drastischen Rückgang der durch Infektionserkrankungen bedingten Todesfälle von ca. 800 auf unter 50 pro 100 000 Einwohner.

Die wesentlichen Ursachen für den auch in anderen aufstrebenden Industrienationen $\mathrm{zu}$ verzeichnenden Rückgang der Bedrohung durch Infektionskrankheiten waren kluge und konsequent umgesetzte politische Entscheidungen zur Stärkung der Gesundheitssysteme. Die politischen Entscheidungen führten z.B. zur Einrichtung von Ministerien für Gesundheit und zur Schaffung von kommunalen Gesundheitsämtern mit starken Kompetenzen und guter personeller Ausstattung. Dies waren Voraussetzungen für die flächendeckende Implementierung effektiver Maßnahmen zur Seuchen- und Infektionsprävention - wie der Verbesserung der sanitären Situation durch die Schaffung von Abwasserversorgungssystemen, der Chlorierung des Trinkwassers oder das Ausrollen nationaler Impfprogramme etwa gegen Pocken und Polio. In Deutschland haben u.a. Ärzte und Forscherpersönlichkeiten wie Rudolf Virchow, Emil von Behring, Robert Koch, Ernst von Bergmann und Paul Ehrlich wesentlich zu dieser Erfolgsgeschichte beigetragen. Erhebliche Investitionen in die Infektionsforschung führten zu effektiven Impfstoffen und antiinfektiv wirksamen Substanzen wie Sulfonamiden, Penicillin und weiteren Antibiotika. Dass vier der ersten fünf Nobelpreise für Medizin und bis 1960 noch zwölf weitere Nobelpreise für Forschungen auf den Gebieten der Infektionserkrankungen und Immunologie vergeben wurden, belegt 


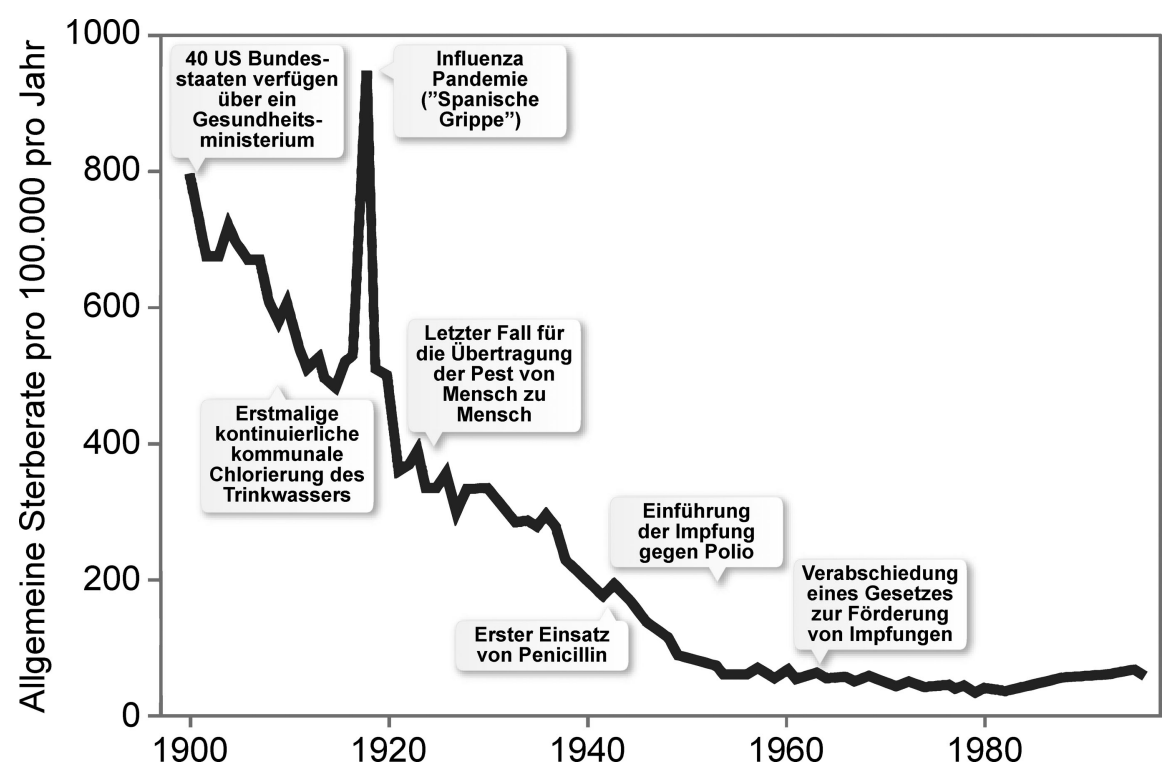

Abb. 1: Trends bei der Entwicklung der Gesamtsterblichkeit infolge von Infektionskrankheiten in den USA im Zeitraum 1900-1997, adaptiert nach Armstrong et al. 1999

die hohe Priorität, die dem Kampf gegen Infektionen und Seuchen von Seiten der Wissenschaft, Politik und Gesellschaft in diesem Zeitraum eingeräumt wurde. Wie selbstverständlich die Notwendigkeit einer engen Kooperation zwischen Medizin und Politik zu dieser Zeit war, lässt sich an den folgenden Zitaten von Rudolf Virchow (1821-1902) ablesen: „Medizin ist eine soziale Wissenschaft und Politik ist nichts Anderes als Medizin im Großen“ und „Medizin hat die Verpflichtung, das Problem zu benennen, die Politiker und die praktischen Anthropologen müssen die Mittel für die aktuelle Lösung des Problems finden“ (Schadewaldt 1972). Die Richtigkeit dieser Auffassung bestätigt sich derzeit im Kampf gegen die COVID-19-Pandemie u. a. in Deutschland in geradezu beeindruckender Weise. Die Folgen von zu späten bzw. falschen politischen Entscheidungen und der mangelnden, verzögerten Umsetzung von medizinischen und wissenschaftlichen Erkenntnissen sind in einigen Ländern wie den USA, Brasilien und auch England für jedermann ersichtlich. Das Beispiel der Folgen des schwedischen Sonderwegs zeigt, wie wichtig auch die Richtigkeit der wissenschaftlichen Evidenz für die getroffenen Maßnahmen ist. 


\section{Ein zweischneidiges Schwert: Der erfolgreiche Kampf gegen die Infektionskrankheiten und seine Folgen für die weitere Entwicklung der Gesundheitssysteme}

Die im Kampf gegen Infektionskrankheiten erzielten Erfolge trugen erheblich zu der trügerischen Annahme bei, dass Infektionskrankheiten endgültig besiegt seien. Der gesundheitspolitische Fokus verlagerte sich vor allem in den westlichen Industrienationen, aber auch bei der WHO, zunehmend auf nicht-übertragbare Erkrankungen. Beflügelt wurde dieser Paradigmenwechsel nicht nur durch das temporäre Niederzwingen der Infektionserkrankungen, sondern auch durch die daraus resultierende gestiegene Lebenserwartung, die $\mathrm{zu}$ einer Erhöhung des Anteils älterer Menschen in der Bevölkerung führte. Diese demographische Entwicklung ging mit einer Zunahme von Herzkreislauferkrankungen, Krebserkrankungen und weiteren sog. Zivilisationserkrankungen wie Diabetes und Adipositas einher. Schnell bildeten sich neue medizinische Fachgesellschaften, die sich auf ebendiese Krankheitsbilder fokussierten und in enger Kooperation mit der Pharmaindustrie den Prioritätenwechsel im Gesundheitswesen beschleunigten. Starke Unterstützung fand dieser Prioritätenwechsel hin zu den nicht-übertragbaren Krankheiten zusätzlich durch Patienteninteressengruppen. In den Vereinigten Staaten forderten zum Beispiel Krebspatienten in den 1950er und 1960er Jahren angesichts der zu diesem Zeitpunkt de facto kaum bestehenden Behandlungsmöglichkeiten ein „Manhattan Project“ und einen „Moon shot for cancer“. Sie postulierten: „For any illness to rise to political prominence, it needed marketing [...]. A disease needed to be transformed politically before it could be transformed scientifically“ (Mukherjee 2010). Mit diesem Vorgehen erreichten sie Gesetzesänderungen, die zur Bereitstellung erheblicher finanzieller Forschungsmittel und z. B. auch zu einer Neuausrichtung und Umstrukturierung des National Institute of Health (NIH) führten. Die beeindruckende Reduzierung der Sterblichkeit und die Linderung der Folgen von Krebs und von chronischen Erkrankungen bestätigen die zweifelsfrei große Nützlichkeit dieser Entwicklung. 


\section{Ursachen und Folgen der Marginalisierung der Infektionsmedizin und des Öffentlichen Gesundheitsdienstes}

Mit diesem fundamentalen Prioritätenwechsel - weg von den übertragbaren, hin zu den nicht-übertragbaren Krankheiten - ging eine fatale Marginalisierung der Infektionsmedizin und des Öffentlichen Gesundheitsdienstes einher, die vor der Phase der ausgeprägten Differenzierung der Medizin in einzelne Fachgebiete noch eine zentrale Rolle einnahmen. Im Ergebnis dieser Entwicklung verloren in den meisten Ländern die verbliebenen Infektiologen die Behandlungshoheit für die Therapie stationärer Patienten mit Infektionen. In der Regel verfügten sie über keine eigenen Bettenstationen, und an Infektionen erkrankte Patienten wurden von den Ärzten der zahlreichen anderen neuen, meist organbezogenen und hoch spezialisierten Fachdisziplinen mitbehandelt.

Für Deutschland waren die Auswirkungen dieses Prioritätenwechsels besonders gravierend. Hier behielt die Infektionsmedizin im Rahmen dieses Differenzierungsprozesses nur noch den Status einer Subspezialität bzw. Zusatzbezeichnung ohne eigenständige Facharztanerkennung. Die mit diesem Statusverlust einhergehende geringere Attraktivität der Infektionsmedizin für den medizinischen Nachwuchs ist sicherlich ein wesentlicher Grund dafür, dass es 1.) im Vergleich zu den USA und Schweden in Deutschland, bezogen auf die Bevölkerungszahl, nur etwa ein Viertel an Infektionsmedizinern bzw. klinischen Mikrobiologen gibt, dass 2.) in Deutschland derzeit nur drei Universitätsklinken über eigenständige Lehrstühle für Infektionsmedizin verfügen, 3.) nur eine Minderheit von Krankenhäusern mit Ärzten ausgestattet ist, die über eine ausreichende Qualifikation für Infektionsmedizin, Mikrobiologie und Hygiene verfügen, während 4.) die Mehrheit der deutschen Krankenhäuser keine eigene Mikrobiologie vorhalten. Im Öffentlichen Gesundheitsdienst der Bundesrepublik arbeiten schätzungsweise nur ca. 2.500 Ärztinnen und Ärzte und viele Stellen können mangels Bewerber nicht besetzt werden. Die fehlende Attraktivität, in diesem Bereich tätig zu sein, resultiert aus dessen Geringschätzung und Vernachlässigung im Rahmen der studentischen Ausbildung, der unzureichend verstandenen Bedeutung des Öffentlichen Gesundheitsdienstes bei der Politik und in der Gesellschaft und nicht zuletzt auch aus der im Vergleich zu anderen Arztgruppen geringeren Vergütung. 


\section{Folgen der Struktur- und Qualitätsprobleme des deutschen Gesundheitswesens}

Die Marginalisierung der Infektionsmedizin und die Vernachlässigung des Öffentlichen Gesundheitsdienstes vollzogen sich völlig unbemerkt und unkommentiert von den sogenannten Organen der Selbstverwaltung des Gesundheitswesens - d.h. u.a. der Deutschen Krankenhausgesellschaft, den Kassenärztlichen Bundesvereinigungen der Ärzte und Zahnärzte und dem Spitzenverband der Krankenkassen, die seit 2004 im Gemeinsamen Bundesausschuss (G-BA) zusammengeführt sind. Dieses höchste Organ der Selbstverwaltung im deutschen Gesundheitswesen hat die Entscheidungskompetenz für die Weiterentwicklung und Qualitätskontrolle des deutschen Gesundheitswesens.

Unbemerkt bzw. ohne Reaktion blieb diese Entwicklung auch bei den für das Gesundheitswesen letztverantwortlichen Aufsichtsinstanzen, dem Bundesgesundheitsministerium, den entsprechenden Ministerien auf Ebene der Bundesländer und den politischen Parteien. Dies änderte sich auch nicht durch das Memorandum für einen Nationalen Sepsisplan, der bereits im Jahr 2013 die Leitung des Bundesgesundheitsministeriums (BMG) auf die gesundheitsökonomische und humane Dimension des Problems hinwies. In diesem Memorandum wurde von Fachexperten aufgezeigt, dass in Deutschland jährlich 15.000 - 20.000 Todesfälle durch Sepsis vermeidbar wären. Dies ist auch deshalb bemerkenswert, weil dieses Memorandum die Unterstützung von zahlreichen medizinischen Fachgesellschaften, Betroffenenorganisationen, dem Präsidenten der Leopoldina, dem Robert Koch-Institut, dem Friedrich-Loeffler-Institut und den Repräsentanten weiterer Forschungseinrichtungen hatte. Die Forderung nach einem Nationalen Aktionsplan blieb unerfüllt, selbst nachdem 2017 anlässlich der Verabschiedung der WHO-Resolution „Improving the Prevention, Recognition, Diagnosis and Clinical Management“" (World Health Organization 2017) ein aktualisiertes 2. Memorandum unter der Schirmherrschaft des Aktionsbündnisses für Patientensicherheit an den neuen Bundesgesundheitsminister gerichtet wurde (Sepsis-Stiftung et al. 2017).

Die WHO-Sepsisresolution war mit Unterstützung des Bundesministeriums für Gesundheit (BMG) und der Bundesregierung verabschiedet worden. Sie hebt hervor, dass die Mehrzahl der Sepsistodesfälle durch eine Verbesserung der Prävention, Früherkennung und des Sepsismanagements vermeidbar ist. Die nationalen Mitgliedsländer der World Health Assembly werden deshalb in dem Dokument dringend dazu aufgerufen, den Kampf gegen Sepsis in ihre nationalen Gesundheitsstrategien aufzunehmen. Inzwischen gibt es in mindestens 15 Ländern entsprechende nationale Strategien, u. a. in Australien, England, Frankreich, 
Irland, Schweden, Saudi-Arabien, dem Sudan und den USA. In Deutschland ist dies jedoch drei Jahre nach Verabschiedung der Resolution noch immer nicht der Fall.

Ein vom Thüringer Sozial- und Gesundheitsministerium initiierter und von der Gesundheitsministerkonferenz der Länder (GMK) im Juni 2018 einstimmig verabschiedeter Beschluss, in dem die Leitung des BMG aufgefordert wird, am Robert Koch-Institut eine Expertenkommission zur Umsetzung der Forderungen der WHO Resolution zur Sepsis einzusetzen, wurde seitens des Gesundheitsministers mit dem Hinweis abgelehnt, dass dies Aufgabe der Länder sei. Lediglich der G-BA hat auf Antrag der Patientenvertreter im Gremium 2018 beschlossen, ein Verfahren $\mathrm{zu}$ eröffnen, mit dem das Ziel verfolgt werden soll, dass die Sepsissterblichkeit ein für alle Krankenhäuser verbindlicher und zu veröffentlichender Qualitätsindikator wird. Die durchschnittliche Dauer von derartigen Verfahren beträgt jedoch ca. 8 Jahre.

Als Folgen dieser Fehlentwicklungen seien beispielhaft aufzuzählen:

- Die Impfrate gegen die saisonale Grippe beträgt in Deutschland bei über 65Jährigen nur 31,4\%, in Australien, England und den USA aber 74\%, 71\% bzw. 69\%. Ähnlich verhält es sich bei Senioren in Bezug auf die Impfung gegen Pneumokokken: In Deutschland sind 35\% geimpft, in Australien, England und den USA 64\%,70\% und 56\% (Fleischmann-Struzek et al. 2018).

- Nach einer repräsentativen Umfrage bei über 60-Jährigen wussten nur 17\% der Befragten, dass eine Impfung gegen Sepsis schützen kann; 23\% glaubten, Sepsis sei Folge einer allergischen Reaktion, 30\% vermuteten fälschlicherweise, dass Sepsis in erster Linie durch sogenannte multiresistente Killerkeime ausgelöst werde, und die Mehrheit glaubte zu Unrecht, dass ein roter Streifen am Arm Hauptsymptom einer Sepsis sei (Eitze et al. 2018).

- Die Begriffe Sepsis bzw. „Blutvergiftung“ kommen in der Gesundheitsberichterstattung des Bundes und der Länder nicht vor. In die Kommunikation der Bundeszentrale für gesundheitliche Aufklärung (BzgA) wurden die Begriffe erst 2021 aufgenommen.

- Im Rahmen eines Qualitätsverbesserungsprojekts zur Optimierung der Sepsiserkennung und -behandlung, an dem sich 40 Krankenhäuer (Bloos et al. 2017) beteiligten, zeigte sich, dass nur ein Drittel der Patienten rechtzeitig, d.h. innerhalb der ersten Stunde nach Diagnose, das Antibiotikum erhielt, während sich durch jede Verzögerung der antibiotischen und chirurgischen Therapie das Sterblichkeitsrisiko erhöht.

- Die Krankenhaussterblichkeit bei Sepsis ist in Deutschland erheblich höher als in vergleichbaren Industriestaaten, gleichzeitig fällt deren Rückgang deutlich geringer aus (siehe Abb. 2). 
- Die Chance, eine Sepsis zu überleben, hängt auch vom Krankenhaus ab, in dem ein Patient behandelt wird: Die risiko-adjustierte Krankenhaussterblichkeit variiert zwischen deutschen Krankenhäusern um den Faktor 2 (siehe Abb. 3).

\begin{tabular}{|c|c|c|c|}
\hline Zeitraum & \multicolumn{2}{|c|}{ Reduktion der Sterblichkeit } & relative Reduktion \\
\hline $2000-2012$ & $35,0 \%$ & $18,5 \%$ & ca. $47 \%$ \\
\hline $2000-2012$ & $45,5 \%$ & $32,1 \%$ & ca. $29 \%$ \\
\hline 2009-2014 & $39,9 \%$ & $23,2 \%$ & ca. $41 \%$ \\
\hline 2009-2014 & $47,8 \%$ & $41,7 \%$ & ca. $12 \%$ \\
\hline
\end{tabular}

Abb. 2: Vergleich des Sterblichkeitsrückgangs bei Patienten mit Sepsis mit Organversagen zwischen Deutschland, Australien, Großbritannien und den USA. In Deutschland zeigte sich in einem vergleichbaren Zeitraum eine geringere Reduktion als in anderen Ländern; hierzulande mit 41,7\% deutlich über dem Niveau anderer Länder, adaptiert nach: Fleischmann-Struzek et al. 2018

Die aufgezeigten Unterschiede in den Überlebensraten bei Sepsis zwischen einzelnen Ländern unterstützen die These: „Sepsis ist Spiegel der Qualität eines Gesundheitswesens" (N. Yamamoto, Assistent Director General der WHO (World Health Organization 2018)).

Die relativ hohe Sepsissterblichkeit in Deutschland zeigt, dass die Qualität eines Gesundheitswesens nicht nur von den dort investierten Ressourcen abhängt. Kein Land der Welt verfügt z. B. über mehr Kapazitäten für die besonders teuren Intensivtherapiebetten als Deutschland - diese sind für die Behandlung von Patienten mit Sepsis sehr wichtig. Doch obwohl Deutschland bei den Kosten für das Gesundheitssystem an der Spitze und $40 \%$ über dem europäischen Durchschnitt liegt, nimmt es bei der Qualität - gemessen an der Zahl der vermeidbaren Todesfälle - im europäischen Vergleich nur einen Mittelplatz ein. Dabei sind die vermeidbaren Todesfälle durch Sepsis in dieser Statistik noch gar nicht berücksichtigt. Wie die Sepsis ist auch der Herzinfarkt eine Erkrankung, die als zeitkritischer Notfall behandelt werden muss. Hier ist Deutschland mit einer 30-Tagesterblichkeit von über $8 \%$ eines der Schlusslichter. Bei den vier europäischen Staaten mit der geringsten 30-Tagesterblichkeit liegt diese bei nur 4\% bzw. deutlich niedriger (European Commission 2020). Auch bei der Behandlung des 
Sepsissterblichkeit im Deutschen Qualitätsbündnis Sepsis (Jahr 2018)

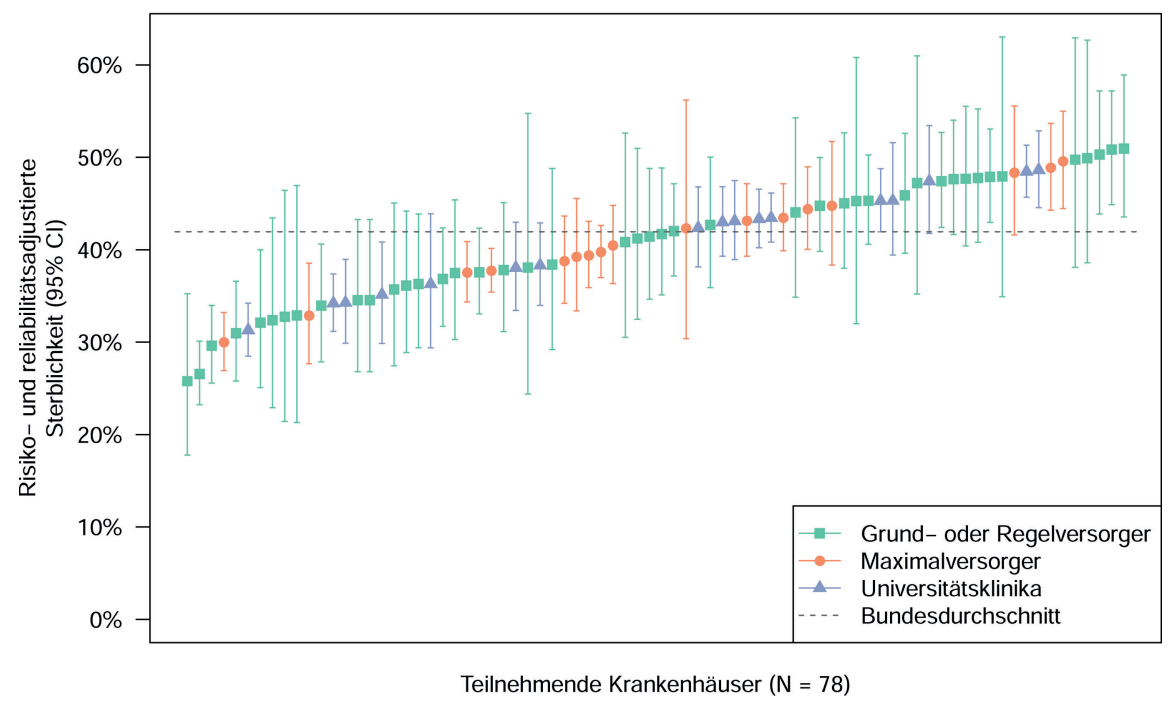

Abb. 3: Vergleich der risikoadjustierten Krankenhaussterblichkeit von Patienten mit Sepsis mit Organversagen im Jahr 2017 zwischen den am Deutschen Qualitätsbündnis Sepsis teilnehmenden Krankenhäusern und dem deutschen Durchschnitt. Bei den teilnehmenden Krankenhäusern findet sich eine große Schwankungsbreite hinsichtlich der Sepsis-Krankenhaussterblichkeit (Rüddel et al. 2018).

Herzinfarkts gibt es in Deutschland kein Ressourcenproblem: In Deutschland werden nahezu doppelt so viele Herzkatheteruntersuchungen pro Kopf der Bevölkerung wie in Österreich durchgeführt (Muhlberger et al. 2020); dort ist die Herzinfarktsterblichkeit interessanterweise um $25 \%$ niedriger. Da es also nicht an Ressourcen mangelt, sind die eklatanten Unterschiede bei den Überlebensraten wohl am ehesten auf Probleme bei der Vorbeugung (durch ungenügende gesundheitliche Aufklärung) sowie auf struktur- und qualitätsbedingte Mängel bei der Therapie zurückzuführen. Es ist belegt, dass bei Sepsis und Herzinfarkt jede Verzögerung der Behandlung zu einer Verschlechterung der Überlebensrate führt. 


\section{Belege für die Effektivität von Qualitätsverbesserungsmaßnahmen auf nationaler und Einrichtungsebene}

Wie schnell in anderen Ländern mit nichtstaatlichen und staatlichen Gesundheitssystemen Erfolge bei der Reduzierung der Sepsissterblichkeit erreicht wurden, ist beispielhaft für den amerikanischen Bundesstaat New York und Irland belegt (Health Service Executive Ireland 2019; Kahn et al. 2019; Levy et al. 2018; New York State Department of Health 2020).

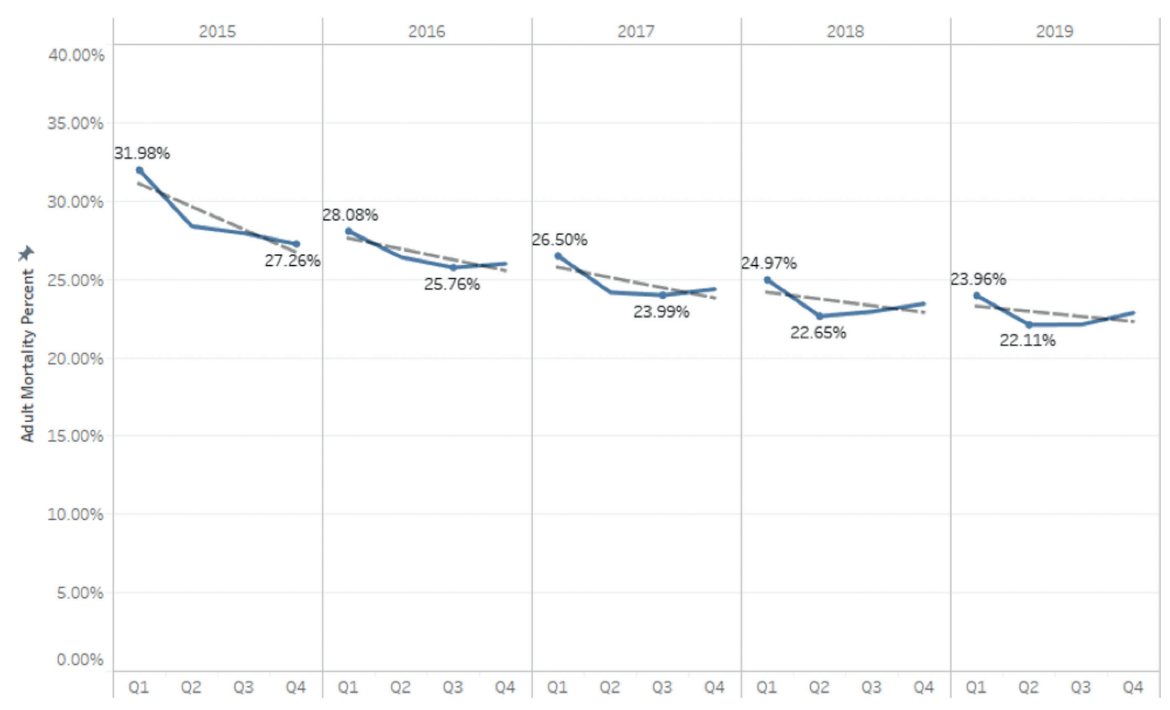

Abb. 4: Verlauf der risiko-adjustierten Krankensterblichkeit nach Initiierung von verbindlichen Sepsis-Protokollen für alle Krankenhäuser im Staat New York. Grafik mit freundlicher Genehmigung von Marcus Friedrich, New York State Department of Health

In Deutschland gibt es derzeit mit dem Universitätsklinikum Greifwald bisher nur eine Klinik, die im Rahmen einer mehrjährigen Qualitätsverbesserungsinitiative über ein Reduzierung der 90-Tagesterblichkeit von 64\% auf $45 \%$ berichtet (Scheer et al. 2017). 


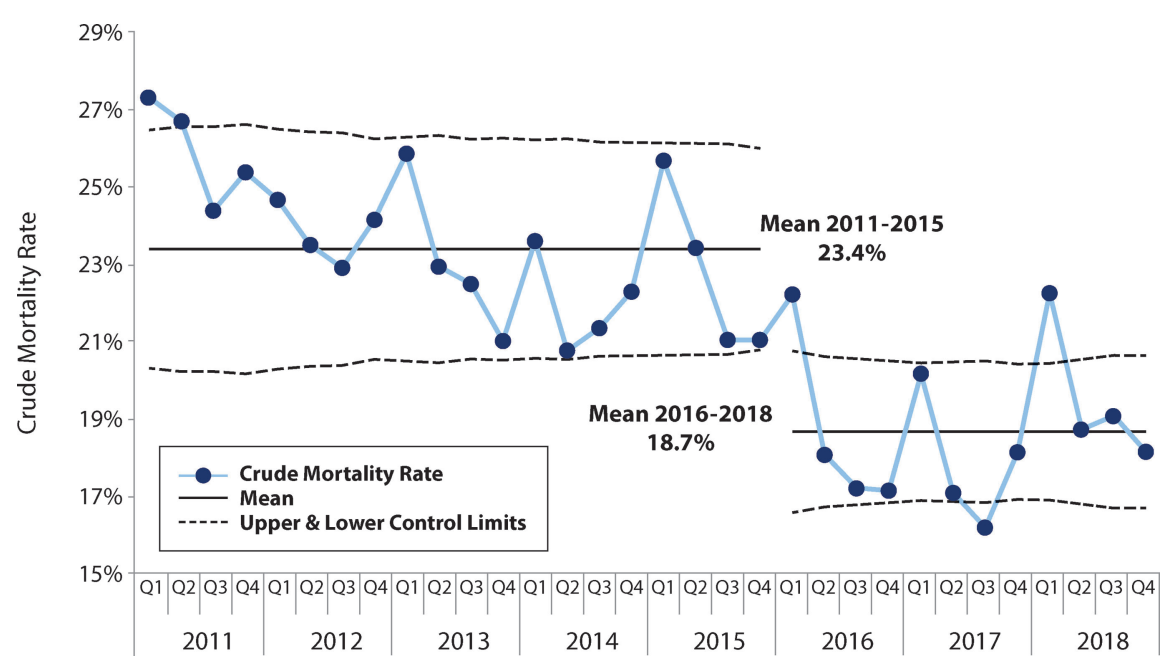

Abb. 5: Vergleich der Sterblichkeitsraten von Sepsispatienten vor und nach Initiierung einer nationalen Aufklärungskampagne in Irland, die Teil der National Clinical Guideline: Sepsis Management (Health Service Executive Ireland 2019, S. 16) war.

\section{Forderungen an die Politik und die Entscheidungsträger im Gesundheitswesen}

Zeitnah:

- Aufklärung der Bevölkerung über Vorbeugemaßnahmen und die Früherkennung von Sepsis (Abb. 6) sowie die Einstufung der Behandlungsnotwendigkeit als Notfall (wie bei Herzinfarkt und Schlaganfall) durch die BZgA. Da sich $70-80 \%$ der zu einer Sepsis führenden Infektionen außerhalb des Krankenhauses entwickeln, ist die Bevölkerungsaufklärung von besonderer Relevanz. Dazu soll ein Sonderetat in Höhe von jährlich 13 Millionen Euro bereitgestellt werden. Als Vorbild sei hier der Sonderetat für die sehr effektive Aufklärung der Bevölkerung über sexuell übertragbare Krankheiten genannt.

- Systematische Aufklärung von Familien mit Neugeborenen und Kindern über die Alarmsymptome von Sepsis sowie das Angebot einer jederzeit erreichbaren Notfallnummer, analog zu Großbritannien, wo dies bereits Standard ist.

- Verpflichtung der Krankenhäuser zur Weiterentwicklung der bestehenden Reanimationsteams zu Medizinischen Notfall Teams und Einführung von verpflichtenden Screeningtools zur Früherkennung vital gefährdeter Patienten in den Notaufnahmen und den stationären Bereichen. 
- Verbindliche Schulung des ärtlichen und pflegerischen Personals im stationären und ambulanten Bereich, z.B. im Hinblick auf Frühwarnzeichen, analog zu der Umsetzung etwa in Großbritannien: National Early Warning Scores (NEWS) (Royal College of Physicians 2017).

- Bundesweite Hinzufügung der Sepsis zu den Tracerdiagnosen für die Rettungsleitstellen.

- Etablierung von Mindeststandards für die fachliche Qualifikation im Rahmen der Anstrengungen des BMG zur Verbesserung der Qualität ambulanter Notfallmedizin durch Schaffung von integrierten Notfallzentren durch den Gesetzgeber (Bundesministerium für Gesundheit 2020).

- Etablierung und Finanzierung eines Deutschen Sepsis Qualitätszentrums in Analogie zum Nationalen Referenzzentrum für Nosokomiale Infektionen durch das BMG.

- Optimierung der Sepsis- und Erregerdiagnostik, die wie für alle anderen Notfalldiagnosen in den Krankenhäusern rund um die Uhr zur Verfügung stehen muss, außerdem der Aufbau einer zeitnahen Erregerdiagnostik im ambulanten Bereich.

- Umsetzung von Maßnahmen zur Förderung der Attraktivität der Infektionsmedizin, Hygiene und des Öffentlichen Gesundheitsdienstes.

Mittelfristig:

- Umsetzung der Forderungen aus dem Memorandum für einen Nationalen Sepsisplan unter der Moderation einer hochrangigen Repräsentantin bzw. eines Repräsentanten des BMG mit Einbeziehung der Länder und des RKIs. Das Ziel sollte hier sein, die sepsisspezifischen Forderungen unter einem Dach mit den bestehenden nationalen Anstrengungen zur Infektionsprävention bzw. zum Infektionsschutz und in Bezug auf Antibiotikaresistenz zusammenzufassen und in einer Deutschen Infektionskontroll- und Managementstrategie zusammenzuführen und aufeinander abzustimmen. So können unnötige Doppelstrukturen vermieden und die vielfältig vorhandenen Synergien zwischen diesen eng verknüpften Themengebieten maximiert werden. Eine wichtige Erstmaßnahme für diesen Schritt wäre die Fortsetzung des Runden Tisches mit den Unterzeichnern des Memorandums und den wichtigen im G-BA vertretenen Interessengruppen unter der Ägide des BMG/RKI und unter Einbeziehung der Repräsentanten der Länder. 


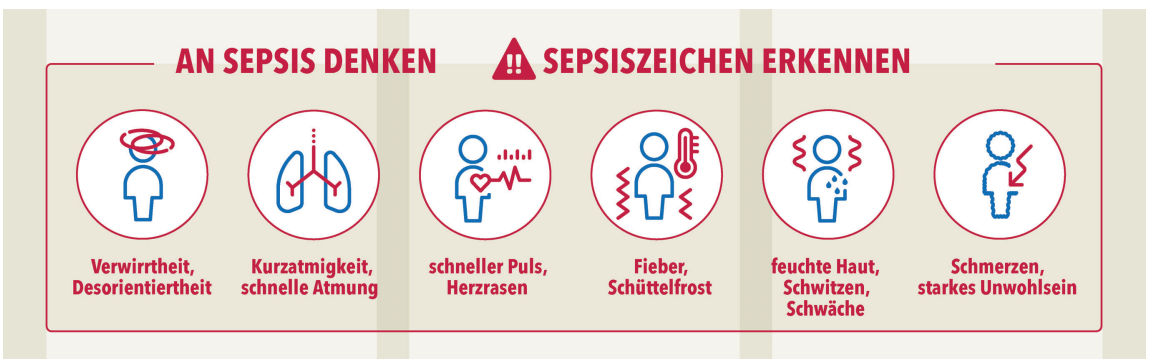

Abb. 6: Die aufgeführten Symptome können darauf hinweisen, dass sich aus einer unkomplizierten Infektion eine lebensbedrohliche Sepsis entwickelt. Fieber alleine, d.h. ohne das Vorliegen eines der anderen Symptome, spricht nicht für eine Sepsis. Mit der Zahl der aufgeführten Symptome steigt die Wahrscheinlichkeit für die Diagnose Sepsis stark an. Auch Symptome wie Durchfall oder Krampfanfälle, die anderweitig nicht erklärbar sind, können für eine Sepsis sprechen. (Quelle: Robert Koch-Institut)

\section{Schlussfolgerungen}

Wenn die COVID-19-Pandemie überwunden sein wird, sollten die immensen humanen und ökonomischen Folgen von Infektionskrankheiten sowie die offensichtlichen Qualitätsdefizite und die mangelnde Kosteneffizienz des deutschen Gesundheitswesens Anlass für einen Dialog über notwendige Strukturveränderungen sein. In diesem Prozess sollte es nicht nur darum gehen, wie die marginalisierte Infektionsmedizin und der Öffentliche Gesundheitsdienst gestärkt werden können. Sondern darüber hinaus gilt es die grundsätzlichere Frage zu klären, ob die Qualitätssicherung, Kosten- und Strukturentwicklung des Gesundheitswesens auch weiterhin primär den im G-BA durch Partikularinteressen und Interessenskonflikten belasteten Organen der Selbstverwaltung im Gesundheitswesens alleine überlassen bleiben können. Die Zusammensetzung des G-BA führt oft zu viel zu langwierigen Entscheidungsprozessen und nicht immer ausreichend am Gemeinwohl und Patienteninteressen orientierten Entscheidungen. Dreh- und Angelpunkt des dringend nötigen Systemwandels und Umbaus des Gesundheitswesens muss die Patientensicherheit und die Einbeziehung von Patientenerfahrungen in die Weiterentwicklung von Arbeits- und Kommunikationsprozessen im ambulanten und stationären Bereich sein. Dies bedeutet auch die Notwendigkeit eines erheblichen Ausbaus der Sicherheits- und Qualitätskultur bei den Anbietern von Gesundheitsleistungen. Diese sind derzeit noch weit entfernt von den nationalen und internationalen Standards, die z.B. in der Luftfahrtindustrie oder bei der Verkehrssicherheit gelten und die dort durch Aufsichtsbehörden streng kontrolliert werden. 
Die vorbildliche und vertrauensstärkende Interaktion zwischen Politik, Aufsichtsbehörden, Wissenschaft sowie den Akteuren im Gesundheitswesen und Gesellschaft vor dem Hintergrund der COVID-19-Pandemie in Deutschland war die zentrale Voraussetzung dafür, dass Deutschland zu den Ländern gehört, die hinsichtlich der Zahl der zu beklagenden Todesfälle relativ gut dasteht. Diese positive kollektive Erfahrung, zusammengenommen mit den aus dieser tiefgreifenden Krise zu ziehenden Lehren, bietet einen guten Anknüpfungspunkt für die Fortführung der von verschiedenen Seiten angestoßenen Debatte über die Notwendigkeit einer tiefgreifenden Reform des deutschen Gesundheitswesens.

\section{Literatur}

Armstrong, Gregory L./Conn, Laura A./Pinner, Robert W. (1999): „Trends in Infectious Disease Mortality in the United States during the 20th Century“. In: Jama 281(1), S. 61-66.

Bloos, Frank/Ruddel, Hendrik/Thomas-Ruddel, Daniel et al. (2017): „Effect of a Multifaceted Educational Intervention for Anti-infectious Measures on Sepsis Mortality: A Cluster Randomized Trial“. In: Intensive Care Med 43(11), S. 1602-1612.

Buchman, Timothy G./Simpson, Steven Q./Sciarretta, K. L. et al. (2020): „Sepsis Among Medicare Beneficiaries: 1. The Burdens of Sepsis, 2012-2018“. In: Crit Care Med 48(3), S. $276-288$.

Bundesministerium für Gesundheit (2020): „Reform der Notfallversorgung“. https://www. bundesgesundheitsministerium.de/notfallversorgung.html, besucht am 5.1.2021.

Eitze, Sahra/Fleischmann-Struzek, Carolin/Betsch, Conrnelia et al. (2018): „Determinants of Sepsis Knowledge: A Representative Survey of the Elderly Population in Germany“. In: Crit Care 22(1), S. 273.

European Commission (2020): „Country Health Profiles“. European Commission. https://ec. europa.eu/health/state/country_profiles_en, besucht am 5.1.2021.

Fleischmann-Struzek, Carolin/Mikolajetz, Antje/Schwarzkopf, Daniel et al. (2018): „Challenges in Assessing the Burden of Sepsis and Understanding the Inequalities of Sepsis Outcomes between National Health Systems: Secular Trends in Sepsis and Infection Incidence and Mortality in Germany“. In: Intensive Care Medicine 44(11), S. 1826-1835.

Health Service Executive Ireland (2019): National Sepsis Report 2018. https://www.hse.ie/eng/ about/who/cspd/ncps/sepsis/resources/national-sepsis-report-2018.pdf, besucht am 8.4.2020.

Kahn, Jeremy M./Davis, Billie S./Yabes, Jonathan G. et al. (2019): „Association between State-mandated Protocolized Sepsis Care and In-hospital Mortality among Adults with Sepsis“. In: Jama 322(3), S. 240-250.

Levy, Mitchell M./Gesten, Foster C./Phillips, Gary S. et al. (2018): „Mortality Changes Associated with Mandated Public Reporting for Sepsis. The Results of the New York State Initiative“. In: American Journal of Respiratory and Critical Care Medicine 198(11), S. 1406-1412. 
Muhlberger, Volker/Kaltenbach, Lalit/Bates, Katie et al. (2020): „Cardiac Catherization in Austria: Results from the Austrian National Cardiac Catheterization Laboratory Registry (ANCALAR) 2012-2018“. In: Wien Klin Wochenschr 132(3-4), S. 79-89.

Mukherjee, Siddhartha. (2010): The Emperor of All Maladies: A Biography of Cancer. New York, NY: Scribner.

New York State Department of Health (2020): „Sepsis Overview“. https://www.health.ny.gov/ diseases/conditions/sepsis/\#rpts, besucht am 5.1.2021.

Rhee, Chanu/Dantes, Raymund/Epstein, Laura et al. (2017): „Incidence and Trends of Sepsis in US Hospitals Using Clinical vs Claims Data, 2009-2014“. In: Jama 318(13), S. 1241-1249.

Royal College of Physicians (2017): „National Early Warning Score (NEWS) 2“. https://www. rcplondon.ac.uk/projects/outputs/national-early-warning-score-news-2, besucht am 18.3.2018.

Rudd, Kristina E./Johnson, Sahra C./Agesa, Kareha M. et al. (2020): „Global, Regional, and National Sepsis Incidence and Mortality, 1990-2017: Analysis for the Global Burden of Disease Study“. In: Lancet 395(10219), S. 200-211.

Rüddel, Hendrik/Schwarzkopf, Daniel/Fleischmann, Carolin et al. (2018): „Sepsis - ein lange unterschätztes und vernachlässigtes Problem“. In: Franz Dormann/Jürgen Klauber/Ralf Kuhlen (Hrsg.): Qualitätsmonitor 2018. Berlin: Medizinisch Wissenschaftliche Verlagsgesellschaft, S. 121-140.

Schadewaldt, Hans (1972): „,Die Politik ist nichts weiter als Medizin im Großen‘: Die Wissenschaftstheorie bei Rudolf Virchow - Erste Fortsetzung (Teil 2)“. In: Dtsch Arztebl 69(36), S. $2302-2303$.

Scheer, Christian S./Fuchs, Christian/Kuhn, Sven-Olaf et al. (2017): „Quality Improvement Initiative for Severe Sepsis and Septic Shock Reduces 90-Day Mortality“. In: Critical Care Medicine 45(2), S. 241-252.

Sepsis-Stiftung/Institut für Hygiene und Umweltmedizin/Deutsche Gesellschaft für Infektiologie (2017): Nationaler Sepsisplan: Handlungsfelder und Maßnahmen. https:// www.sepsis-stiftung.eu/wp-content/uploads/1/2018/11/2018_04_01_Memorandum_ Sepsisplan_gesamt.pdf, besucht am 6.1.2020.

Singer, Mervyn/Deutschman Clifford, Christopher S./Seymour, C. W. et al. (2016): „The Third International Consensus Definitions for Sepsis and Septic Shock (Sepsis-3)“. In: Jama 315(8), S. 801-810.

World Health Organization (2017): „World Health Assembly 70, Resolution 70.7.: Improving the Prevention, Diagnosis and Clinical Management of Sepsis“. Genf: World Health Organization, http://apps.who.int/gb/ebwha/pdf_files/WHA70/A70_R7-en.pdf, besucht am 12.1.2021.

World Health Organization (2018): „WHO Sepsis Technical Expert Meeting - Meeting Report“. World Health Organization. https://www.who.int/servicedeliverysafety/areas/sepsis_ meeting-report-2018.pdf, besucht am 5.1.2021.

World Health Organization Headquarters (2020): „Clinical Management of COVID-19“. World Health Organization. https://www.who.int/publications/i/item/clinical-management-ofcovid-19, besucht am 5.1.2021. 